\title{
CARDIAC INVOLVEMENT IN EOSINOPHILIC GRANULOMATOSIS WITH POLYANGIITIS - A CHALLENGING CASE REPORT
}

\author{
Larissa Maria Oliveira Gonzaga ${ }^{1, \star}$, Ana Luisa Bagno de Almeida ${ }^{1}$, Aurivan Essado Dantas ${ }^{1}$, Carolina Ruas Freire Santos ${ }^{1}$, Anna \\ Carolina Faria Moreira Gomes Tavares ${ }^{1}$, Leandro Augusto Tanure ${ }^{1}$, Gilda Aparecida Ferreira ${ }^{1}$
}

1.Universidade Federal de Minas Gerais, Belo Horizonte (MG), Brazil.

*Corresponding author: gonzagamlarissa@gmail.com

\section{BACKGROUND}

Eosinophilic granulomatosis with polyangiitis (EGPA) is the least frequent antineutrophil cytoplasmic antibody (ANCA)associated vasculitis, but is the most affected by heart disease. The endocardium, myocardium, pericardium and coronary arteries can be affected, with cardiomyopathy as the main manifestation. As most patients can be asymptomatic, cardiac involvement is often underdiagnosed, although it is the major cause of death in patients with this disease.

\section{CASE REPORT}

A 68-year-old female patient, diagnosed with EGPA (difficult-to-treat asthma, chronic sinusopathy and multiple mononeuritis) at 50, ANCA negative, taking methotrexate and prednisone, already treated with cyclophosphamide. History of long-term smoking, systemic arterial hypertension, diabetes mellitus, and heart failure with reduced ejection fraction (EF 37\%) of undefined etiology. In February 2021, she evolved with atypical chest pain, fatigue and worsening of baseline dyspnea. A new transthoracic echocardiography (TTE) presented with significant reduction in the $\mathrm{EF}(21 \%)$ and increasing segmental deficit. Cardiac magnetic resonance imaging (MRI) showed EF $17 \%$, multiple areas of akinesia and fibrosis, with delayed transmural contrast enhancement, nontypical involvement of atherosclerosis, no signs of active myocarditis. Also, a negative troponin and a normal cardiac catheterization. Thus, we considered progression of heart disease due to EGPA's asymptomatic cardiac activity over the years, at the moment with no further evidence of disease activity.

\section{CONCLUSION}

Although EGPA is related with increased cardiovascular risk due to inflammation in the vessel wall and premature atherosclerosis, the main mechanism of cardiac injury is eosinophilic infiltration. Coronary vasculitis is rarely seen. Studies show that in case of myocardial injury related to eosinophils there is a progression from acute necrosis to fibrosis and any delay in treatment may result in irreversible myocardial damage. The initial tests are electrocardiogram and TTE. Cardiac MRI is especially important for asymptomatic patients and PET-CT is useful in case of delayed MRI enhancement, when it helps to distinguish between inflammation and fibrosis. Myocardial biopsy is the gold standard test. The treatment is immunosuppression with high-dose corticosteroid and cyclophosphamide or rituximab. Rarely, heart transplantation is indicated. In the case presented, because no myocardial activity was identified, only damage, we chose not to immunosuppress. Heart transplantation is under discussion. Cardiac involvement is the main predictor of mortality in EGPA (50\%). These patients usually have frequent relapses, negative ANCA and eosinophilia. Because the prevalence in patients in sustained remission of the disease is high, early diagnosis and treatment can be difficult and need close attention.

\section{KEYWORDS}

ANCA-associated vasculitis, Heart disease, Eosinophils. 